\title{
Study on the Measurement Model of Person-Job Fit Degree Based on Polynomial Regression
}

\author{
Lei LUO ${ }^{\mathrm{a}}$, Yuanyuan LIU ${ }^{\mathrm{b}, *}$
}

School of Economics and Management, Beijing Jiaotong University, Beijing 100044, China

a19120703@bjtu.edu.cn, b17120702@bjtu.edu.cn

${ }^{*}$ Corresponding author

Keywords: person-job fit, measurement model, polynomial regression

\begin{abstract}
Traditional person-job fit degree measurement models such as PSIs (profile similarity indices) or weighted difference scores cannot highlight the role of different competency. In this paper, the polynomial regression method is introduced to construct the quadratic polynomial regression measurement model of person-job fit degree, which improves the method of difference scores and solves the problem of the original models. Finally, this paper introduces the general application process of the quadratic polynomial regression measurement model of person-job fit degree.
\end{abstract}

\section{Introduction}

In an increasingly competitive market economy, what companies need is not to spend huge sums of money to recruit the best employees, but to recruit the most suitable employees, that is, to achieve person-job fit. How to accurately and effectively achieve person-job fit has obviously become an urgent problem to be solved.

To achieve person-job -fit, it is necessary to quantitatively measure person-job fit degree. Only when we can measure the specific degree of fit between employees and positions, can we choose the most suitable person. In recent years, scholars have proposed many fit degree measurement models based on different theories. Among them, PSIs (profile similarity indices) and weighted difference scores (which combines analytic hierarchy process and fuzzy comprehensive evaluation method) are currently used more models. However, there is no consensus on which model can most accurately measure the person-job fit degree. It is necessary to review these models and analyze the problems of them. Based on it, this paper will try to introduce the polynomial regression method to improve the problem existing in the original model.

\section{Analysis of traditional fit degree measurement model}

\subsection{PSIs (profile similarity indices)}

The earliest and most widely used methods for measuring the person-job fit degree is the method of PSIs (profile similarity indices). This method is not only used in the person-job fit measurement, but also widely used in the study of organizational consistency. In this method, the two sets of measurement data, including job requirements and personal competency scores, are combined into one score to represent the overall person-job fit degree ${ }^{[1]}$. One of the most important part of the PSIs model is the method of difference scores. This method is to calculate the difference scores between the competency requirement of the job and the score of the individual competency, so that the difference score is used to represent the fit degree. The implicit meaning is that the smaller the difference scores between the competency requirement of the job and the score of the individual competency, the more fit. Among them, for how to use the difference scores to represent the fit degree, the mathematical formula can be used flexibly according to different situations to calculate the difference sum representing different meanings, such as $\mathrm{D}^{1}$--directly calculating the difference sum, $|\mathrm{D}|$--calculating the absolute value of the difference sum, $\mathrm{D}^{2}$--calculate the of the sum of the squares of the differences, D--calculate the square of the difference and then re-opening, and the Mahalanobis 
distance-- M's D that takes the weights of the different competencies into account. The specific formula can be seen in Table 1 .

Table1 Difference Score Formula

\begin{tabular}{cc}
\hline Index & formula \\
\hline$D^{2}$ & $\sum\left(x_{i}-y_{i}\right)^{2}$ \\
$D$ & $\sqrt{\sum\left(x_{i}-y_{i}\right)^{2}}$ \\
$|D|$ & $\sum\left|x_{i}-y_{i}\right|$ \\
$D^{1}$ & $\sum\left(x_{i}-y_{i}\right)$ \\
$M^{\prime}$ s D & $\sqrt{\left(x_{i}-y_{i}\right)^{\prime} S^{-1}\left(x_{i}-y_{i}\right)}$ \\
\hline
\end{tabular}

\subsection{Weighted difference scores}

The above method ignores the fact that the degree of importance of different competency to person-job fit is different. Therefore, in order to highlight the differences in the role of different competency in the measurement of fit, many scholars try to empower each competency, so that the important competency's weight is great, and the unimportant competency' weight is small. Because the standard value of different competency of the job and the empowerment of each competency are subject and difficult to define, many studies in China combine the methods of analytic hierarchy process $(A H P)$ and fuzzy comprehensive evaluation to construct the measurement model of person-job fit degree, in order to reduce the influence of subjective factors and random factors on the calculation of the fit degree, so that the results are more objective and effective.

\subsection{Problem to be solved}

We conclude that the person-job fit degree measurement model constructed by the analytic hierarchy process and the fuzzy comprehensive evaluation method is an improvement of the difference-score method. The basic principle is to calculate the difference scores between the person and the job to represent the fit degree. However, weights can not fully highlight the important competency. The difference in the contribution of each competency to the person-job fit does not come from the different weights given in the calculation, but on the overall variance ${ }^{[2]}$. The weight itself cannot determine the contribution of each competency to the final fit degree, but the weight can change the contribution of the original competency to the degree of matching. Therefore, it is necessary to introduce a new method to improve the original difference scores model to measure the person-job fit degree.

\section{Introduction of polynomial Regression}

\subsection{Polynomial regression}

Polynomial is the basic concept in algebra. It is an algebraic expression obtained by a variable called a definite element and a constant called a coefficient by a finite number of addition and subtraction, multiplication, and power of a natural power. Polynomial regression is a regression analysis method that studies the polynomial relationship between a dependent variable and one or more independent variables. It is widely used in the mapping of input and output of fitting data in statistical research.

In fact, in recent years, more and more scholars in organizational consistency studies have tried to use polynomial regression to try to quantitatively analyze the impact of the consistency of people and organizations on the relevant performance of employees such as satisfaction, performance, turnover rate, pressure, etc., and get some new discoveries. ${ }^{[3]}$ In the case that there are serious problems in the PSIs model and the weighted fit degree measurement model, we will try to introduce the polynomial regression method to promote the study on the measurement of person-job fit. Next, we will analyze the feasibility of introducing a polynomial regression method to the measurement model of person-job fit degree. 


\subsection{Feasibility of Polynomial Regression}

As we all know, the study on person-job fit is exactly the study on consistency between the person and the job. Therefore, the traditional methods of measuring fit are based on the difference between the person and the job, that is, the difference score between the person and the job represents the fit degree. For example, for the difference score $\mathrm{D}^{1}, \mathrm{x}_{\mathrm{i}}$ which represents the individual's i-th competency score, and $\mathrm{y}_{\mathrm{i}}$ represents the score of the $\mathrm{i}$-th competency of the job requirement, there are calculation models as follows:

$$
\mathrm{D}^{1}=\sum\left(x_{i-} y_{i}\right)
$$

$\mathrm{D}^{1}$ is used to represent the fit degree, that is, $\sum\left(\mathrm{x}_{\mathrm{i}}-\mathrm{y}_{\mathrm{i}}\right)$ is used to represent the fit degree. Then we add the coefficient $b_{1}$ to the original formula, and add the constant term $b_{0}$ and the random error term $\varepsilon$ to construct the equation:

$$
\mathrm{Z}=b_{0}+b_{1} \sum\left(x_{i-} y_{i}\right)+\varepsilon \text {. }
$$

Obviously, the equation dependent variable $\mathrm{Z}$ can also represent the fit degree. In addition, adding the parameter before the original matching degree formula represents that the various competent quality features are given the same weight contribution to the matching degree. We may wish to expand the equation:

$$
\mathrm{Z}=b_{0}+b_{1} x_{1-}-b_{1} y_{1}+b_{1} x_{2}-b_{1} y_{2}+\ldots+b_{1} x_{n-} b_{1} y_{n}+\varepsilon .
$$

Among them, $\mathrm{n}$ represents the number of competent qualities required for the job. After the expansion, it is not difficult to find that the fit degree $\mathrm{Z}$ is substantially determined by the $2 \mathrm{n}$ variables of $x_{i}$ and $y_{i}$, that is, the matching degree $\mathrm{Z}$ is a function of $x_{i}$ and $y_{i}$. However, when we directly use the difference score $\mathrm{D}^{1}$ to represent the fit degree, we directly impose some constraints on the function to be solved. The coefficients of each competency variable are the same as $b_{1}$ and the competency variables of the individual and the job are equal in magnitude and opposite in direction $\left(b_{1}\right.$ and $-b_{1}$, respectively). Obviously, these imposed constraints are not reasonable, because the degree of contribution of each competency to the degree of fit is not equal. In order to make the constructed fit equation more efficient, we need to remove these constraints:

$$
\mathrm{Z}=b_{0+} b_{1} x_{1+} b_{2} y_{1+} b_{3} x_{2+} b_{4} y_{2+} \cdots+b_{2 n-1} x_{n+} b_{2 n} y_{n+\varepsilon}
$$

So far, we have obtained the unconstrained polynomial regression model of person-job fit degree, which improved by the difference score $\mathrm{D}^{1}$. The model can solve each parameter by regression analysis to obtain the fit function. The fit function is obviously more effective than the difference score $\mathrm{D}^{1}$ to predict the fit degree. It solves the problem that the difference score cannot highlight the important competency, and also solves the problem that the weight value is difficult to observe in the weighted fit degree measurement model because it gives different coefficients for each competency variable, this parameter is not determined by human comparison, but is derived from empirical data through regression analysis, which is of practical significance.

In the same way, we can derive the other polynomial regression models of person-job fit degree, which is improved by the difference scores $\mathrm{D},|\mathrm{D}|, \mathrm{D}^{2}$, etc., and will not be repeated here.

\section{Construction of Quadratic Polynomial Regression Model of Fit Degree}

In the above we have already discussed that it is feasible to introduce a polynomial regression method to the fit degree measurement model. Then the key problem for constructing a polynomial regression model of fit degree is to determine the number and power of the model variables. The number of variables in the model can obviously be determined directly by the number of competent 
qualities required by the job. But the power of the variable is what we need to consider. We must compare the polynomial regression model, the quadratic polynomial regression model, the cubic polynomial regression model, etc. to determine the optimal power degree polynomial regression model of fit degree to most effectively predict the fit degree.

When constructing a polynomial regression model on the organizational consistency study, Edwards JR (1995) found that a polynomial regression equation is insensitive to the variation of the variables on the curve that can be detected by the quadratic polynomial regression equation, and tests that the increment of $\mathrm{R}^{2}$ is significant after adding a quadratic term to the polynomial regression equation. The change brought about by adding three or more high-order items is not significant. ${ }^{[4]} \mathrm{We}$ should choose the quadratic equation when constructing the polynomial regression model of fit degree, because the quadratic equation can fully describe the relationship between each competency and fit degree. The lower order equation can not fully capture their relationship and the higher-order equations can't capture more relationships than quadratic equations can capture. Then the quadratic polynomial regression model of fit degree is as follows:

$$
\mathrm{Z}=b_{0+} b_{1} x_{1+} b_{2} y_{1+} b_{3} x_{1+}^{2} b_{4} x_{1} y_{1+} b_{5} y_{1}^{2}+\ldots+b_{5 k-4} x_{k+} b_{5 k-3} y_{k+} b_{5 k-2} x_{k+}^{2} b_{5 k-1} x_{k} y_{k+} b_{5 k} y_{k}^{2}+\varepsilon(5)
$$

Among them, $x_{i}$ represents the $i$-th personal competency score, $y_{i}$ represents the $i$-th job competency requirement, $b_{i}$ represents the coefficient of the $i$-th term of the polynomial, $\varepsilon$ represents the random error term, and $\mathrm{z}$ represents the fit degree.

When this model is used to measure the fit degree of a specific position, the job competency requirement is constant, that is, $\mathrm{y}_{\mathrm{i}}$ is constant and will not change due to different employees. Therefore, $\mathrm{y}_{\mathrm{i}}$ can be regarded as a constant term. By merging similar items, the formula is:

$$
\mathrm{Z}=b_{0}+b_{1} x_{1}+b_{2} x_{1+}^{2} b_{3} x_{2+} b_{4} x_{2+}^{2} b_{5} x_{3}+b_{6} x_{3+\cdots+}^{2} b_{2 n-1} x_{n+} b_{2 n} x_{n+\varepsilon}^{2} .
$$

\section{Application Process}

Using the polynomial regression to construct the fit degree measurement model is essentially to construct a quadratic function about the competency of the parameters and then use a large number of samples to carry out regression analysis to solve the parameters of the function. In this function, the dependent variable is the fit degree, and the independent variable is the competency. Obviously, through the function, we can get the fit degree of the employee by inputting the corresponding competency scores of the corresponding personnel. Moreover, in the above, it has been proved that the quadratic regression equation of fit degree is more effective and reasonable than the regression equations of other orders. Therefore, we should focus on constructing the quadratic polynomial regression equation in the measure of fit degree. Specifically, there are 4 steps below.

1. Determine the content and number of variables in the polynomial regression model - that is, determine the competency of the job. This step is the premise of measuring the person-job fit degree. Only by determining the variables of the model, we can further solve the fit degree function with parameters. For different positions, the competency is very different. Therefore, to obtain the competency, you can rebuild the specific competence of the post by interviewing, questionnaire and other methods. You can also directly refer to the competency model that has been developed by the predecessors with high reliability and validity to determine the competency of the job to be studied.

2. Obtain a sample of the polynomial regression model - that is, determine the competency score of the sample personnel and the corresponding fit degree. The sample data of course contains the independent variables $\mathrm{x}_{\mathrm{i}}$ which represent the personal competency scores and the dependent variables $y_{i}$ which represent the fit degree. The competency scores can be directly measured through behavioral event interviews, questionnaires, etc., and the $\mathrm{Z}$ value for each person can be represented by his performance score. 
3. The regression analysis and test of the model — that is, solving the quadratic function of fit degree. After obtaining the sample data, we can use most of the data as regression samples and use SPSS and other statistical software for regression analysis to obtain the regression equation coefficients of a certain confidence level. Then, the remaining part of the sample is substituted into the regression equation to check whether the predicted $\mathrm{Z}$ value is consistent with the actual $\mathrm{Z}$ value.

4. Use the fit degree function to measure employee' fit degree. After the above method tests that the predicted fit degree of the regression equation is consistent with the actual fit degree within a certain error range, it indicates that the regression equation can better predict the matching degree according to the individual competency score. Finally, the polynomial equation after regression can be used to measure the fit degree of the target employees.

\section{Summary}

On the basis of reviewing the traditional fit degree measurement models at home and abroad, it is found that the widely used PSIs model or the weighted difference score model based on the method of AHP can not highlight the difference in the role of different competency qualities. Faced with these problems, this paper attempts to introduce the polynomial regression method into the person-job fit degree measurement model and further construct the quadratic polynomial regression equation of fit degree. Finally, this paper summarizes the general process of applying this model to measure the person-job fit degree.

\section{References}

[1] Edwards J R. Problems with the use of profile similarity indices in the study of congruence in organizational research[J]. Personnel Psychology, 1993, 46(3):641-665.

[2] Zhang Xian, Liu Shengyan, Wang Wenguang. Construction and Application of Person-job Fit Model: Taking the Practice of Person-job fit in Company A as an Example[J]. China Human Resources Development, 2014(22): 54-60.

[3] Zong Na. Research on Person-job Fit Evaluation Model Based on Analytic Hierarchy Process and Fuzzy Comprehensive Evaluation Method[J]. China Securities and Futures, 2013(1): 259-260.

[4] Edwards J R. Ten difference score myths. [J]. Organizational Research Methods, 2001, 4(3):265-287.

[5] Hambleton A J, Kalliath T, Taylor P. Criterion-Related Validity of a Measure of Person-Job and Person-Organization Fit[J]. New Zealand Journal of Psychology, 2000, 29(2):80-85.

[6] Kristof - Brown A L, Zimmerman R D, Johnson E C. Consequences of Individuals' Fit at Work: A Meta - Analysis of Person - Job, Person - Organization, Person - Group, and Person - Supervisor Fit[J]. Personnel Psychology, 2010, 58(2):281-342.

[7] Shi Dongfeng. A Review of Competency-Based Research on Person-job Fit[J]. Journal of Social Sciences (New Theory Edition), 2011(1): 59-60.

[8] Wang Qing, Qi Ershi. Measurement of Person-job Fit Based on AHP-Fuzzy Comprehensive Evaluation[J]. Ergonomics, 2007, 13(2): 19-22.

[9] Li Fei. Analysis of the Person-job Fit in Human Resource Management[J]. New West, 2011. 